\title{
Manufacturing of linear variable filters with straight iso-thickness lines
}

\author{
Laetitia Abel-Tiberini, Frédéric Lemarquis, Gérard Marchand, \\ Luc Roussel, Gérard Albrand and Michel Lequime, \\ Institut Fresnel, UMR CNRS 6133 \\ Université Paul Cézanne, domaine universitaire de Saint Jérôme \\ 13397 Marseille Cedex 20, France
}

\begin{abstract}
Linear variables filters are band-pass multilayer coatings manufactured with a thickness gradient that allows a significant wavelength shift of the centring wavelength of the filter according to the point that is illuminated. In case such a filter is associated with a 2D matrix detector in order to form a compact spectrometer, iso-thickness lines must be as straight as possible, perpendicularly to the thickness gradient. To answer this problem, we developed a masking mechanism that combine the classical rotation movement of substrates and a translation movement for the mask, this last movement being induced during the rotation by the mean of a cam. Thickness gradient can be freely adjusted according to specifications, while transverse uniformity is $99.9 \%$.
\end{abstract}

Keywords: Optical coatings, Thickness uniformity, Linear variable filters, Characterization

\section{1) INTRODUCTION}

Given the list of refractive indices and thicknesses $\left(\mathrm{n}_{\mathrm{i}}, \mathrm{d}_{\mathrm{i}}\right)$ of a thin film coating, optical properties can be easily calculated. More precisely, optical properties depend on the ratio of the optical thickness by the wavelength for each layer $\mathrm{n}_{\mathrm{i}}, \mathrm{d}_{\mathrm{i}} / \lambda$. As a consequence, assuming that all materials are dispersion free, multiplying all thicknesses by a constant factor results in a wavelength shift of the optical properties. For most deposition processes this kind of thickness modification naturally occurs over the coating surface due to the non uniform thickness distribution. In most cases, peoples tend to reduce this phenomenon so that the optical properties of the coating are stable all over the surface. On the contrary, we can increase the thickness non uniformity to produce coatings with spatially variable optical properties, according to the point that is illuminated. For example, components with a circular thickness distribution, corresponding to a circular distribution of the optical properties can be useful for laser beam shaping ${ }^{1}$. Another classical example corresponds to linear variable filters which are in fact band-pass filters with a thickness gradient along one direction. Such components can be associated to array or matrix detectors in order to design spectrometers. In that case, instruments are compact, light and at last reliable since there is no moving part. Considering that these qualities are of great interest for space optics, the Paris-Meudon Observatory asked us for the manufacturing of such filters. Typically, filters must be deposited on square substrates, $20 \mathrm{~mm}$ aside, with a centring wavelength changing by at least a factor 2 from one side to the other. The band width of the filter is not a critical point and roughly corresponds to the spectral range covered by the coating divided by the number of pixels of the detector. In order to be compliant with space environment, coatings must be manufactured using a high energy deposition process. In the present case, we decided to use Dual Ion Beam Sputtering (DIBS), mainly for geometrical reasons that will be justified later.

The basic principle for the manufacturing of intended non uniform coatings consists to use mechanical moving masks in the deposition chamber to master the thickness distribution. In that case, the free parameters to adjust the thickness gradient are the mask shape and its trajectory during deposition. This technique can be implemented easily if we consider linear filters for array detectors. On the contrary, considering now a 2D filter for a matrix detector, not only the thickness distribution should correspond to the required thickness gradient along one direction, but iso-thickness lines should be as straight as possible in the perpendicular direction. Such a result is much more difficult to obtain, due the natural thickness non uniformity of the deposition chamber. The goal of this paper is to describe the set of tools we developed to answer this problem. First, we developed a spectrophotometer dedicated for localized measurement so that we are able to

Advances in Optical Thin Films II, edited by Claude Amra, Norbert Kaiser, H. Angus Macleod, Proc. of SPIE Vol. 5963, 59630B, (2005) · 0277-786X/05/\$15 - doi: 10.1117/12.624779 
perform automatic mappings of highly non uniform coatings with a spatial resolution as low as $100 \mu \mathrm{m}$. Second, we developed a numerical model of the deposition process which allowed us both to characterize the shape of the sputtered plum for each material and to calculate the thickness distribution of the coating. At last we developed a mechanism which permits to associate both a rotation and a translation movement for the mask, which permits to achieve linear variable filters with straight iso-thickness lines.

\section{2) DESCRIPTION OF THE MEASUREMENT BENCH}

In this section, we will briefly describe the spectrophotometer we developed especially for the characterization of highly non uniform coatings ${ }^{2}$.

The main problem for the characterization of such coatings is that the wavelength shift of the optical properties across the measurement beam diameter rapidly gives an average result that can be far from optical properties corresponding to a pinpoint measurement. Ideally, since the filter will be associated with a matrix detector, the measurements should be performed with the spatial resolution corresponding to one pixel, and the more convenient is in fact to use directly this matrix detector to perform this measurement ${ }^{3}$. However, this configuration is not very flexible and our goal was to design a bench with specific capabilities for non uniform coatings, but that could be used easily for any kind of reflectance and transmittance measurements, as far as possible. The bench design is illustrated in figure 1.

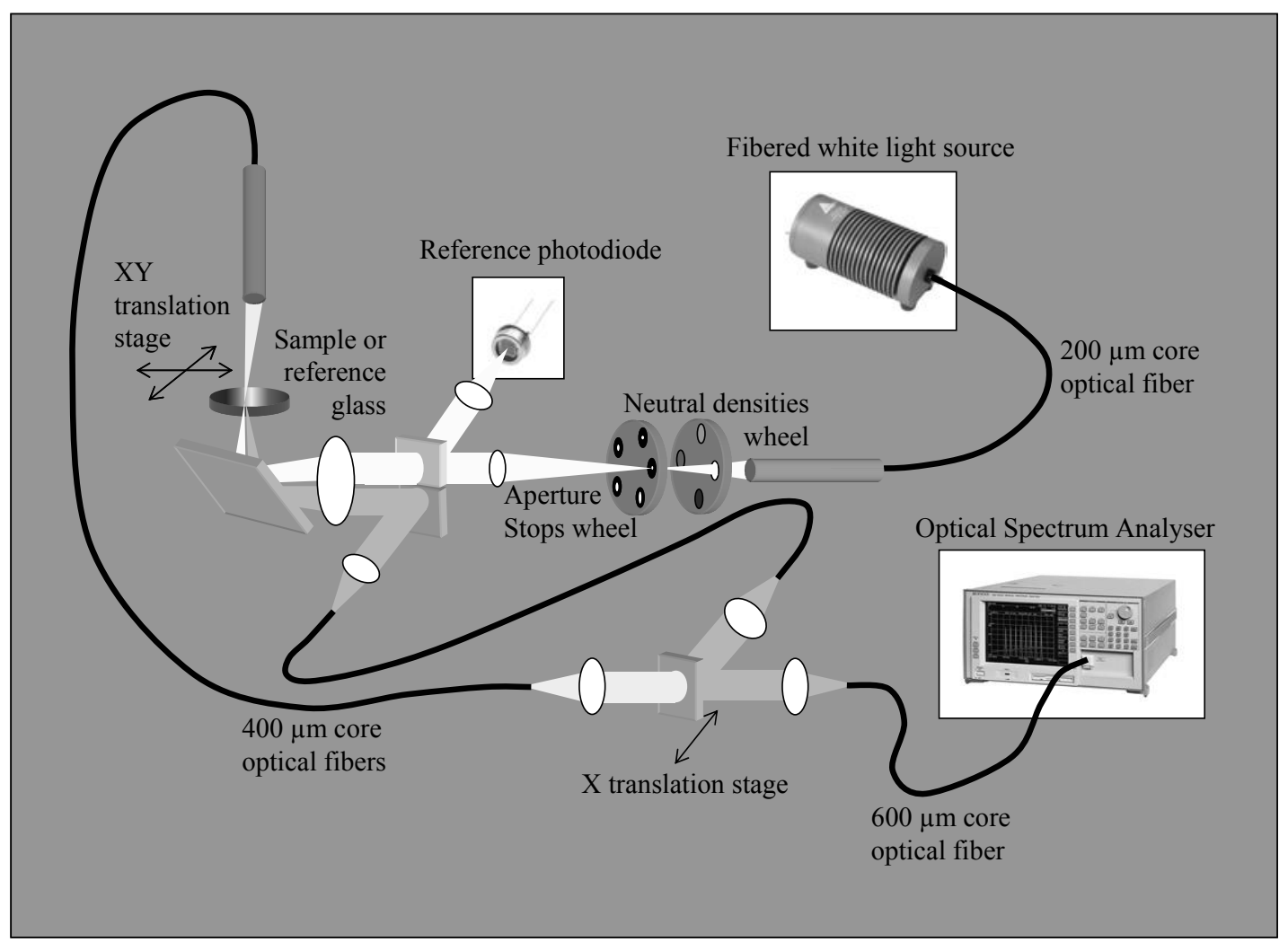

Figure 1: Schematic representation of the measurement bench.

Calibrated apertures permit to define on the sample a probing zone diameter from $100 \mu \mathrm{m}$ to $2000 \mu \mathrm{m}$. Spectral range extends from 400 to $1700 \mathrm{~nm}$. 
At the entrance, the system is illuminated by a quartz-halogen light source coupled in an optical fibre. At the output, the spectral analysis is performed by an optical spectrum analyser operating from 400 to $1700 \mathrm{~nm}$. The sample is placed in between, as well as a set of calibrated apertures that are imaged on the sample surface in order to select the diameter of the measured area (from $100 \mu \mathrm{m}$ to $2000 \mu \mathrm{m}$ ). Two channels are available, either for the reflected or transmitted beams, which are finally connected to the spectrum analyser. Typically, the spectral resolution is between 0.5 and $5 \mathrm{~nm}$, according to the diameter of the optical fibre that is connected to the spectrum analyser and which plays the role of the entrance slit of the monochromator. The sample is motorized along two translations stages so that we can perform automatic mapping on a $60 \times 25 \mathrm{~mm}$ area. The position accuracy is typically $3 \mu \mathrm{m}$.

As an illustration, figure 2 gives the transmittance measured along the thickness gradient on a linear variable filter. According to the position along this axis, we can observe the wavelength shift of the optical properties, due to the thickness gradient. We can also notice a decrease of the band pass transmittance towards shorter wavelengths. Corresponding reflectance measurements show that this phenomenon is not due to absorption. This is in fact the result of both the spatial and spectral integration of the optical properties across the beam diameter and over the spectral resolution of the spectrum analyser.

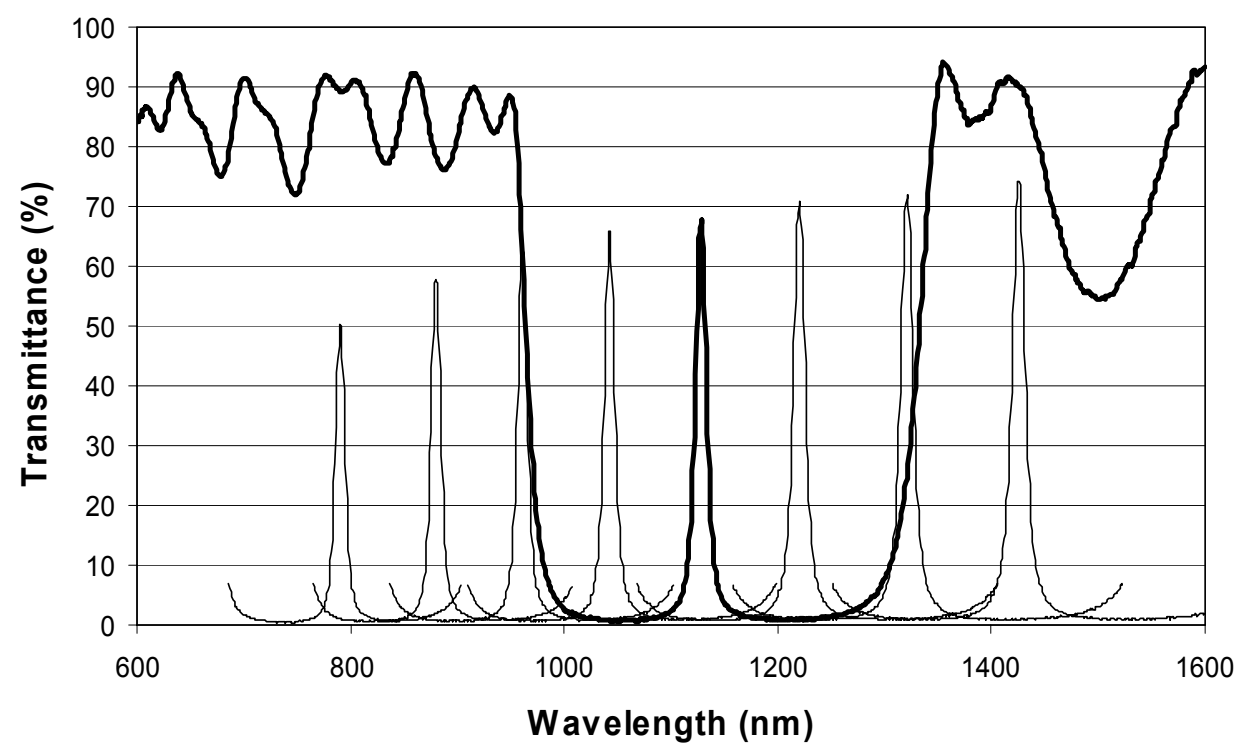

Figure 2: Transmittance profiles measured on a linear variable filter with a $2 \mathrm{~mm}$ step along the thickness gradient. The spot diameter is $200 \mu \mathrm{m}$

As a conclusion, this measurement bench permits us to characterize the thickness gradient of linear variable filters, and its mapping capabilities allow to estimate the curvature of iso-thickness lines.

However, the goal is to produce coatings with the right thickness gradient. This requires finding a correct shape for the mask that will be used during deposition, but first requires the knowledge of the vacuum chamber natural thickness non uniformity. 


\section{3) DEPOSITION PROCESS MODEL}

The deposition process we use is Dual Ion Beam Sputtering, as illustrated in figure 3.

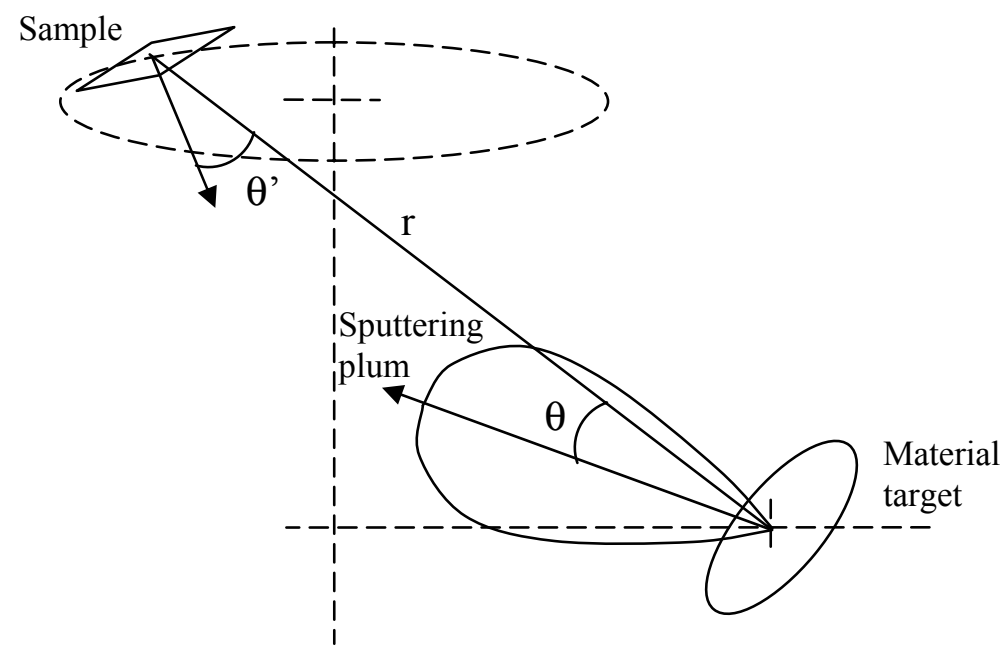

Figure 3: Representation of the deposition process geometry

In order to predict the thickness distribution on the sample, we developed a numerical model based on the classical equation:

$$
t=\int_{\text {sputtering } t \text { arg } e t} \frac{\cos ^{a}(\theta) \cdot \cos \left(\theta^{\prime}\right)}{r^{2}} d s
$$

For a given point of the sample, this equation gives the thickness amount received from the whole target surface. Given a point on the target surface and another one on the substrate, following figure $3, \theta$ is the angle between this line joining these two points and the main sputtering direction while $\theta$ ' is the angle between the line and the normal direction to the sample. ' $r$ ' is the distance between these two points and ' $a$ ' and exponent that represent the sputtering directivity.

Using this equation, we are able to simulate the thickness distribution all over the sample. In case a mask is placed between the sputtering target and the sample, the influence of the mask must be evaluated for each couple of points, in order to take account or not of the corresponding thickness increment. In case the sample is moving, this calculation must be integrated along the whole trajectory.

All the geometrical parameters in this model can be estimated easily. However, several parameters depending on the material are still unknown, such as the main sputtering direction and directivity. The evaluation of these parameters is still under progress. The method we use for this consists to deposit single layers of each material on large static substrates and to optimize these sputtering parameters until a good agreement is reached between the calculated and measured thickness distributions.

However, using typical values for these unknown parameters, this model can be used to simulate the effect of the mask on the thickness distribution, and more precisely on iso-thickness lines curvature. 


\section{4) MECHANISM FOR STRAIGHT ISO-THICKNESS LINES}

In order to increase the coating thickness uniformity, substrates are classically rotating in the upper part of the deposition chamber. Using this configuration, the first linear variable filter we manufactured was produced using a static mask, as illustrated in figure 4 . The outer side of the substrate is hidden half the time behind the mask while the inner side is never, so that the thickness is multiplied by a factor two from one side to the other. This filter was of great interest to test the capabilities of the measurement bench presented in section 2. However, the great disadvantage of this simple masking method is that iso-thickness lines are circular, due to the rotation of the substrate holder. All the points located on the same radius are coated identically.

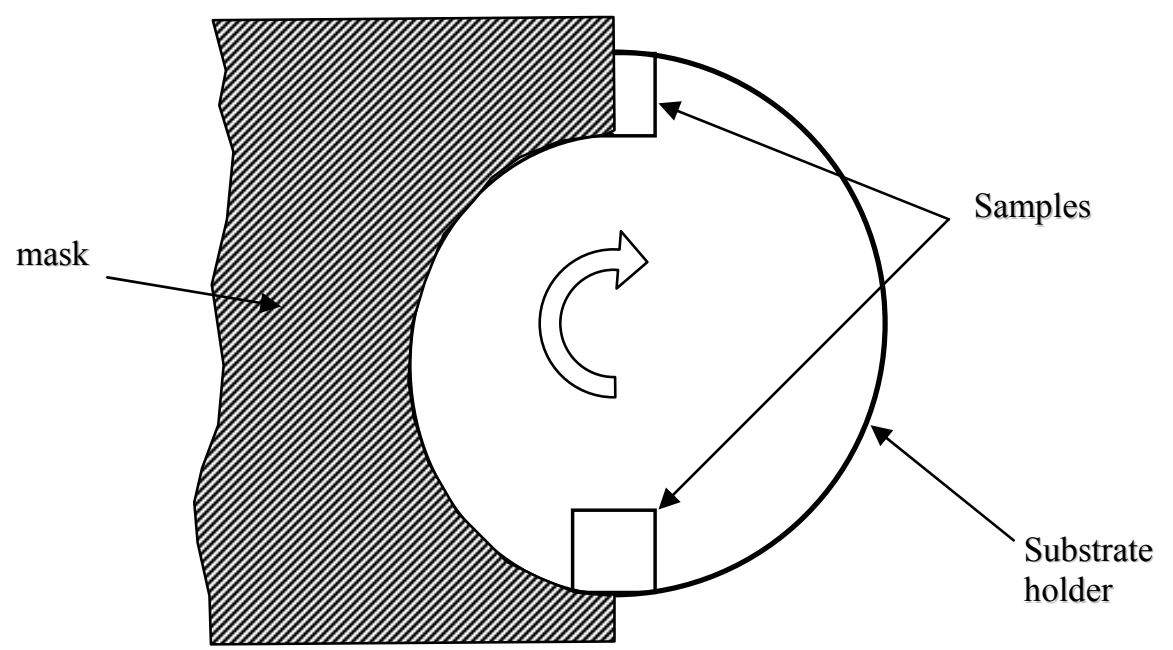

Figure 4: Representation of the masking technique using a static mask and a rotation of the substrates

In order to obtain straight lines, the first idea is to use a static substrate, and a translation movement for the mask. In that case, iso-thickness lines are straight provided that the mask edge is straight and that the natural thickness distribution of the deposition chamber is uniform. One disadvantage of this geometry is that only one filter can be manufacture at a time, unless a mask with a specific movement is developed for each one. To avoid this problem, we imagined a solution that combine both the rotation of the substrate and the translation of the mask. Following figure 5, a translation stage is fixed on the substrate holder and permits to translate the mask just bellow the substrate. And this translation movement is obtained, during the rotation of the substrate by the mean of a cam surrounding the substrate holder. A single substrate is represented on figure 5, but several can be coated at the same time provided that each one is equipped with a mask. However, the great advantage of this system is that the movement, that is to say the cam profile, is the same for all the masks.

Using such a system is not a sufficient condition to obtain straight iso-thickness lines. Let us consider the substrate represented in figure 5, and more precisely the three points $\mathrm{A}, \mathrm{B}$ and $\mathrm{C}$ that are aligned with the mask edge. The goal is to obtain identical thickness for these three points. First, the substrate being in the horizontal plane, and no mask being used for the moment, alls the points located on the same rotation circle receive the same amount of deposited material during one complete revolution.

Due to the deposition chamber non uniformity, the angular distribution of this amount of material that is a periodic and symmetrical function, with a maximum closer to the material target, and a minimum on the opposite side. Such angular distributions curves are given on figure 6 for deferent radius values. Notice that all curves intersect near angles 60 and 300 degrees. 


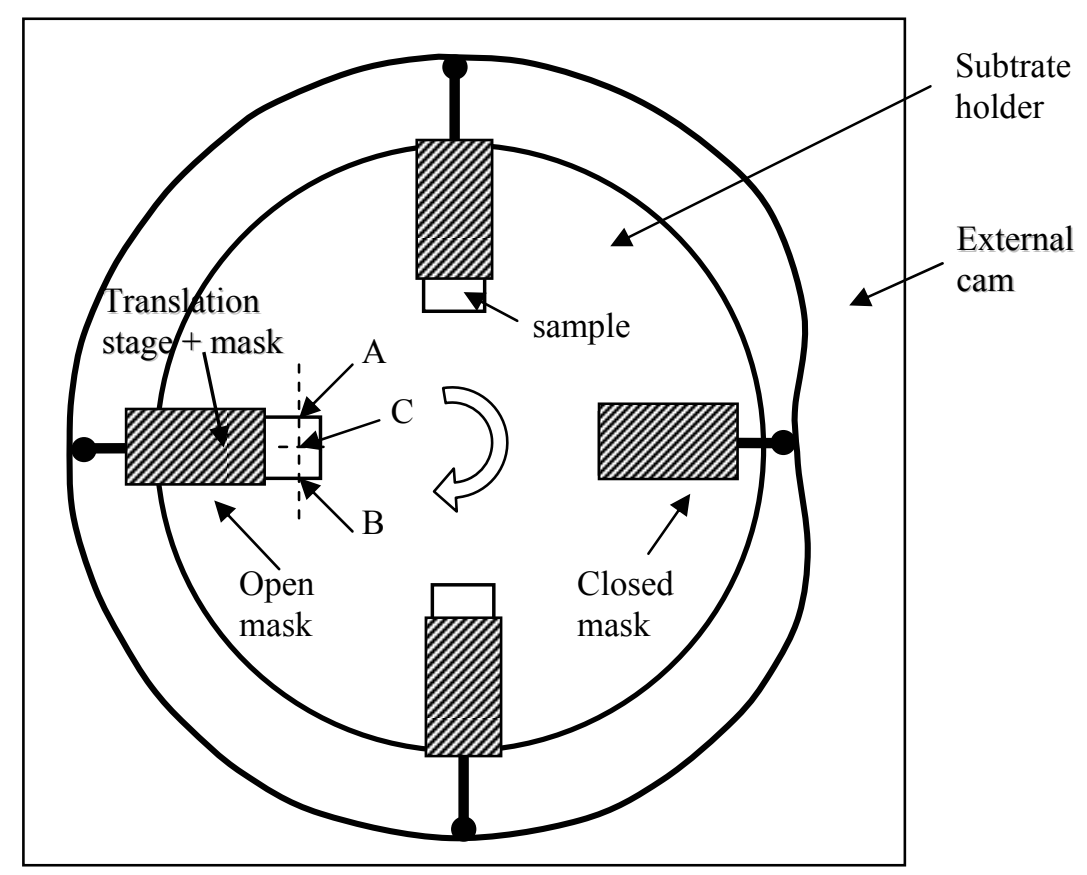

Figure 5: Representation of the masking technique using both a translation of the masks and a rotation of the substrates. The translation movement is given by an external cam during the rotation of the substrate holder.

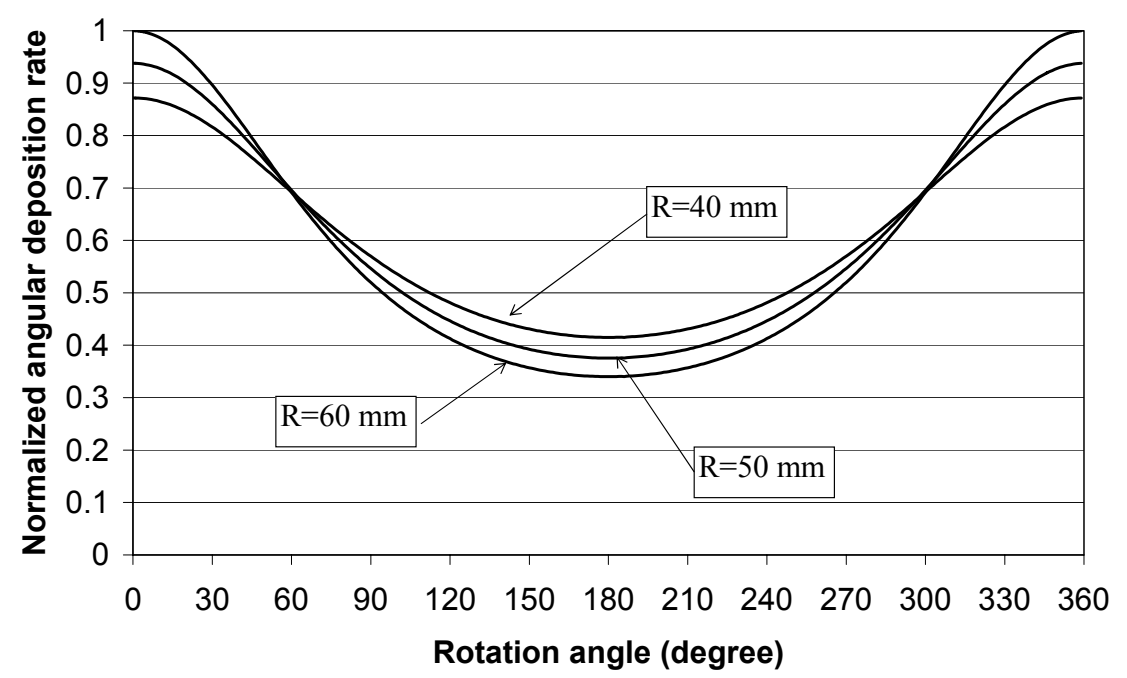

Figure 6: Normalized angular deposition rate for different radius $\mathrm{R}$ on the substrate holder

Points A and B are located on the same radius and thus correspond to one of these curves. However, since these points are angularly separated each point lead to one curve, with the corresponding angular shift as represented on figure 7 . Considering that the radius is $50 \mathrm{~mm}$, the angular separation is about \pm 11 degrees for substrate size of $20 \mathrm{~mm}$. These curves are identical, but simply shifted by the angular distance between the two points. Suppose now that the mask allows the deposition only during the angular sector noted $\Delta \theta 1$. Since the total amount of material deposited during this time is given by the area below these curves, obviously, the two points A and B are not coated identically. 


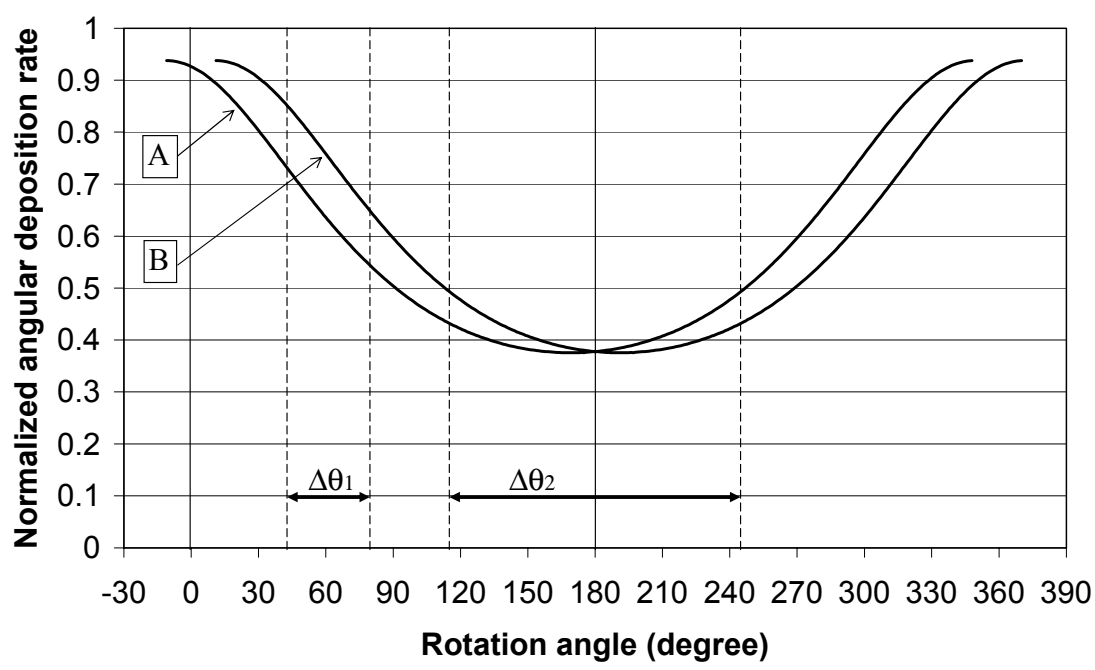

Figure 7: Normalized angular deposition rate for 2 points located on the same radius $(50 \mathrm{~mm})$ but angularly separated $\left( \pm 11^{\circ}\right)$. In order to obtain identical thickness for such points, the mask must opened symmetrically $\left(\Delta \theta_{2}\right)$.

The only solution to avoid this phenomenon is to allow the deposition during an angular sector (noted $\Delta \theta_{2}$ on figure 7) that is centred on the symmetrical axis of the system. In that case, point A receives more deposited material than point B during the first half turn, but this thickness difference is perfectly compensated during the second half turn.

As a consequence, to obtain a symmetrical thickness distribution perpendicular to the main thickness gradient, the cam profile must be symmetrical with its symmetrical axis aligned with the direction defined by the material target and the rotation axis. Notice that the angular sector corresponding to deposition can be split in several sectors, provided that the symmetry is respected. At last, using the same cam for each material, it is therefore necessary that all material sources are located at the same place, which is the case for the DIBS process.

Assuming such geometry, let us now consider point $\mathrm{C}$ which is not located on the same radius. As a result, the angular thickness distribution is not identical for this point, compared to points A and B. Figure 8 gives the angular distribution curves corresponding to points $\mathrm{A}$ and $\mathrm{C}$.

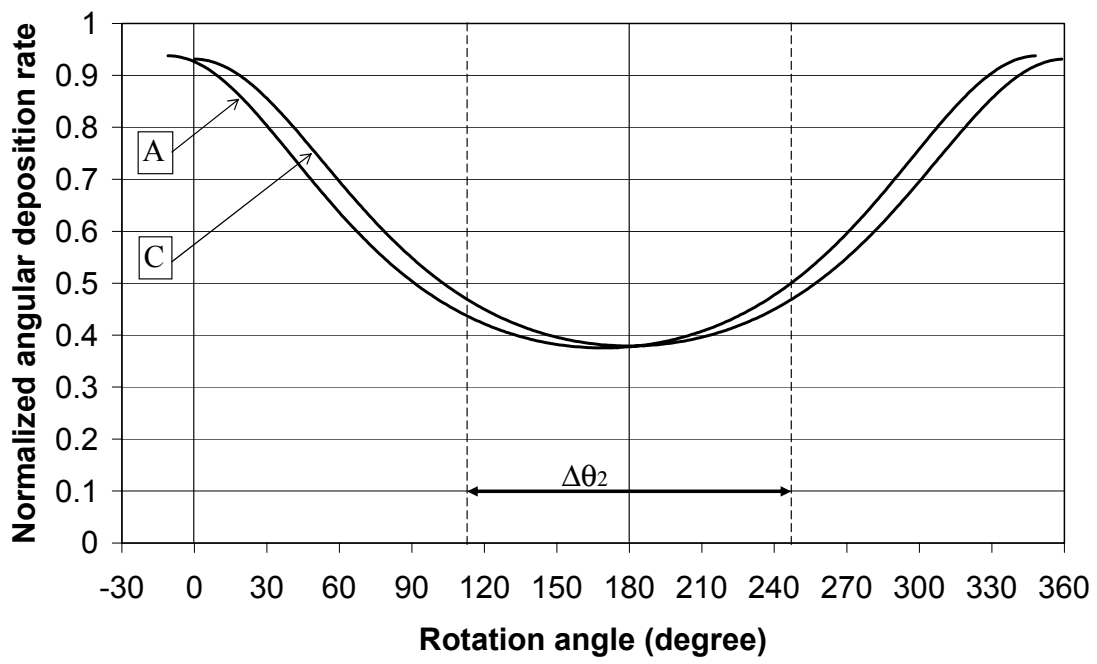

Figure 8: Normalized angular deposition rate for one point located on the substrate symmetrical axis $(\mathrm{C}, \mathrm{R}=49 \mathrm{~mm})$ and a point located on the substrate side.

(A, $\mathrm{R}=50 \mathrm{~mm}$ ). The angular separation is $11^{\circ}$. The thickness mismatch between these points is almost cancelled is the mask is opened symmetrically $\left(\Delta \theta_{2}\right)$ 
As previously, the thickness difference between these points is given by the difference of the areas below the two curves. According to figure 8 where the curves nearly intersect on the symmetry axis, the thickness difference between points A and $\mathrm{C}$ naturally tends to be minimized. Probably the cam profile can improve the result but it seems that only numerical simulations can lead to the optimum shape.

At present time, we are able to simulate the thickness distribution using such a mechanism. As an illustration, figure 9 gives the results for a square substrate, $20 \mathrm{~mm}$ aside, while figure 10 gives the cam profile that was used to perform this calculation. The main result is that the non uniformity in the direction perpendicular to the main thickness gradient is less than $0.2 \%$. This result is as good as the result obtained with a static substrate, located on the opposite side of the target material. Another important fact is that this result is not very sensitive to the sputtering parameters such as sputtering direction and directivity used in the model.

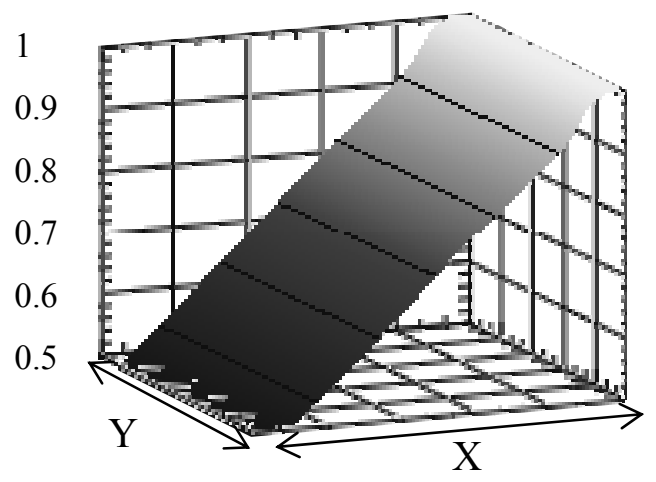

Figure 9: Thickness uniformity calculated with the cam profile given in figure 10. The thickness ration equals 2 while transverse uniformity is better than $99.8 \%$.

Figure 10 Position of the mask edge during the rotation of the substrate holder. The cam profile can be deduced from this curve by simply adding a constant distance.

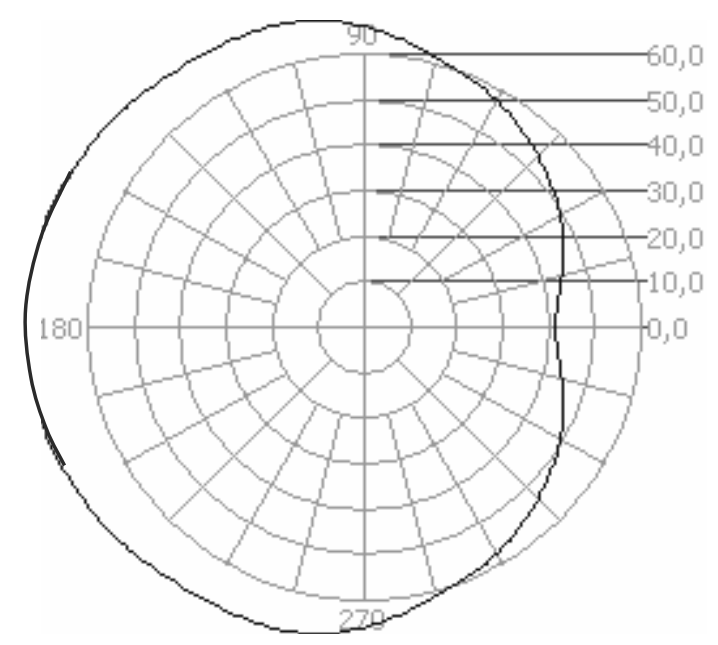

We also developed a prototype of the mechanism, as shown in figure 10. One can recognise two substrates in this photo, with their translation stages and masks, as well as the external cam. We manufactured several samples which were measured using the bench detailed in section 2. A post treatment of the data has been developed to take account of an alignment mismatch between the thickness gradient and the mapping axes. As a final result, we measured perpendicularly to the main thickness gradient a non uniformity of $0.3 \%$, which is compliant with the order of magnitude found with simulations. 


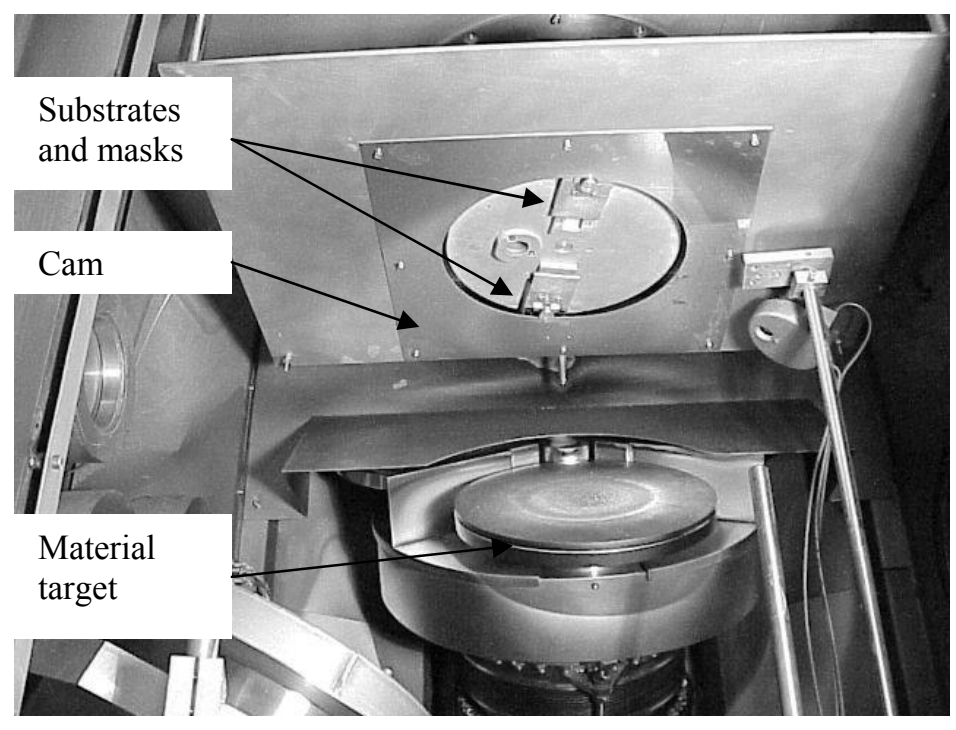

Figure 11: Picture of the deposition plant with the masking mechanism. Notice that the mask is closed near the target while the other is open.

\section{5) CONCLUSION AND PERSPECTIVES}

We have described in this paper all the tools we developed for the manufacturing of linear variable filters. These tools concern optical metrology on very small areas, simulation of the deposition process to take account of its natural non uniformity, and masking mechanism to achieve straight iso-thickness lines. All these tools have been tested and proved their efficiency since the coating uniformity, perpendicularly to the main thickness gradient was measured equal to 0.003 , this value being confirmed by calculations. Notice that the main advantage of the mechanism we developed is that several linear variable filters can be produced at the same time.

Now, in the aim of producing linear variable filters with prescribed optical properties, and using the mechanism we developed, we need to calculate specific cam profiles, taking account of the natural thickness distribution of the deposition process. For this purpose, we decided first to characterize this thickness distribution for each material. This characterization is performed on large static substrates on which single layers are deposited. After a complete mapping of their optical properties, we are able to extract the thickness distribution, and using an optimization procedure, to find the most appropriate values for the sputtering parameters used in our model. This work is still under progress. Second, we developed an optimization procedure to define the cam profile. For this purpose, since this profile can be considered as a $2 \pi$ periodical function, the cam is described by set of Fourier coefficients which are used as free parameters for the optimization. Naturally, the merit function we calculate is related to the thickness gradient along the main filter direction and thickness uniformity perpendicularly to this direction.

This software is now achieved and as soon as the sputtering parameters will be definitely characterized, we will be ready for the manufacturing of linear variable filters that have the required wavelength shift, while offering straight isothickness lines across the main direction.

\section{REFERENCES}

1. Angela Piegari and Gabriele Emiliani "Laser mirrors with variable reflected intensity and uniform phase shift: design process" Applied optics Vol. 32, No. 28,1 October 1993

2. $\quad$ L. Abel-Tiberini, F. Lemarquis, M. Lequime: "Dedicated spectrophotometer for localized transmittance and reflectance measurements", submitted to Applied Optics

3. A.Krasilnikova, A.Piegari, M.Dami, L. Abel-Tiberini, F.Lemarquis, M.Lequime: "Spatially resolved spectroscopy for non-uniform thin film coatings: comparison of two dedicated set-ups", SPIE, Optical Systems Design Jena, Sept 2005. 